\title{
Redundancy allocation in time-varying channels with long propagation delays
}

\author{
Beatrice Tomasi $^{\mathrm{a}, *}$, Daniele Munaretto ${ }^{\mathrm{b}}$, James C. Preisiga, ${ }^{\mathrm{a}, \mathrm{c}}$, Michele Zorzi ${ }^{\mathrm{b}}$ \\ ${ }^{a}$ Applied Oceanic Physics and Engineering, Woods Hole Oceanographic Institution, 266 Woods Hole Rd, \\ Woods Hole, MA (USA) \\ ${ }^{b}$ Department of Information Engineering, University of Padova, Padova, Italy \\ ${ }^{c} J P A n a l y t i c s$ LLC, Falmouth, MA (USA)
}

\begin{abstract}
In this paper, we propose an optimal redundancy allocation over a binary symmetric channel by using recent results on a tight approximation of codeword error probability in the finite blocklength regime. We evaluate the performance of the proposed algorithm over experimental data where slowly time-varying channel conditions were measured. The rationale of this study is to enhance the performance of underwater acoustic communications, for which low bit rates are available. The performance of the proposed scheme is compared with those obtained for a constant rate allocation and a rateless scheme. Results confirm that in the presence of a time-varying channel with long propagation delays, the utilization of a robust codeword is inefficient in terms of bandwidth used and energy per bit. However, as it results in a small number of retransmissions, it also guarantees short delivery delays. Furthermore, in the case where a lower reliability can be tolerated, the proposed scheme is more efficient and also has shorter delivery delays.
\end{abstract}

Keywords: Underwater acoustic communications, redundancy allocation, reliability.

${ }^{*}$ Corresponding author

Email addresses: btomasi@whoi. edu (Beatrice Tomasi), munaretto@unipd.it (Daniele Munaretto), jpreisig@jpanalytics.com (James C. Preisig), zorzi@dei.unipd.it (Michele Zorzi)

Preprint submitted to Journal of Ad Hoc Networks

December 6, 2014

(C) 2015. This manuscript version is made available under the Elsevier user license http://www.elsevier.com/open-access/userlicense/1.0/ 


\section{Introduction}

The recent development and employment of autonomous underwater vehicles, underwater sensors, and acoustic buoys motivate the interest in the design of reliable and energy-efficient underwater acoustic (UWA) communication strategies. Reliability translates into spectral efficiency. Spectral efficiency is important because the achievable data rate scales roughly with available bandwidth, which is a scarce resource in the UWA channel. At the same time, since transmission is the most energy consuming activity of an UWA modem [1, 2], spectral efficiency results in energy savings that are vital to keep the aforementioned devices operational for a longer time, given that both unsuccessful transmissions and unnecessary redundancy are reduced as much as possible.

Following this reasoning, in this paper we pose and solve a simple optimization problem, from which we then design a redundancy allocation scheme for packets transmitted over a time-varying channel with long propagation delays. Throughout this paper, we assume that the bit error process caused by this time-varying channel has a constant probability over the duration of a single packet, but it can change over different packets. Therefore, we indicate as non-stationary conditions those when the crossover probability changes over different packets. In particular, a Binary Symmetric Channel (BSC) model is assumed to be representative for the bit error process at the output of an adaptive equalizer that compensates for time-varying attenuation and intersymbol interference, typical of the underwater acoustic channel. Then, we compute the amount of redundancy that maximizes a metric that combines spectral efficiency and reliability. Consequently, we design a realtime algorithm that computes and allocates the amount of redundancy required to maintain the desired quality of service. Such redundancy 25 is computed based on some channel state information that is estimated from a previously received control message, such as a probing sequence. The performance of the proposed scheme is compared with those obtained for a constant rate allocation and a rateless scheme. Results confirm that in the presence of a time-varying channel with long propagation delays, the utilization of a robust codeword is inefficient in terms of used bandwidth and energy per bit. However, as it guarantees a smaller number of 
retransmissions, it also results in short delivery delays. When a lower reliability can be tolerated, the proposed scheme becomes more efficient and has shorter delivery delays.

As widely investigated in the past few years for terrestrial wireless communications, e.g., see [3, 4, 5, 6, 7], energy saving can be achieved by designing scheduling schemes characterized by low-power and long (low-rate) transmissions, as also proposed in [8]. In addition, these energy-efficient transmission schemes assume capacity achieving codes, which implies longer codewords, especially in poor channel conditions. The relatively low symbol rate supported by the underwater channel along with the decrease in the data rate (bits/symbol) to enable lower power operation and the attempt to get close to capacity at this reduced power level combine to give very long duration codewords. As a result, the channel is time-varying over the duration of a codeword while in the aforementioned literature it is assumed to be time-invariant. This is particularly likely in UWA scenarios, since the propagation of acoustic waves depends on stochastic processes that are in turn time-varying, such as surface waves, currents, and how the sound speed profile is statistically distributed in time and space. In this paper, we move towards a more realistic representation of the time-varying channel by assuming that the channel statistics can vary among different codewords, but it remains stationary during a packet transmission.

In the literature, the problem of reliable UWA communications has been studied, 50 e.g., in [9, 10, 11, 12, 13, 14]. In particular, the authors of [9, 10] investigate the performance of rateless coding schemes used for broadcast communications. In [11], the authors study the tradeoff between delay and reliability in a multi-hop sensor network. The authors of [12] perform a simulation study on the reliability achieved by the proposed Automatic Repeat reQuest (ARQ) technique, tailored to UWA communications. Even though these studies provide insight on the reliability associated to a multi-user channel in different networking scenarios, the presented results are limited to the case of time-invariant channel fading, since only the dependence on distance is considered.

In contrast to this previous work, we focus on the unreliability due to the time variability of the statistics for the underwater acoustic channel. In particular, we design and evaluate a scheme that allocates the redundancy required to protect UWA transmissions, based on some channel state information (CSI). This CSI is estimated at the 
transmitter by decoding a probing sequence piggy-backed in the ACKs/NACKs sent by the receiver to the transmitter, upon the receipt of a packet.

In [14], the authors design a super-Nyquist modulation and rateless coding scheme suitable for doubly-selective underwater acoustic channels. However, they do not explicitly study how to optimally allocate the redundancy to decrease the number of retransmissions. The authors of [15] propose a rateless coding scheme, whose distribution adapts to the fading conditions and which runs based on limited CSI (ACK/NACK) available at the transmitter. They show that this adaptive rateless code outperforms the standard rateless codes in terms of throughput. In this work a similar adaptive coding scheme is considered. However, the proposed scheme consists in adapting the coding rate depending on the time-varying communication perfomance at each single transmission.

The structure of the present paper is summarized as follows. In Sec. 22 we present the considered scenario and the statement of the problem together with some related work. In Sec. 3, three allocation schemes are described: the proposed one, a constant allocation and a rateless scheme. In Sec. 4 , we evaluate the redundancy allocations over experimental data, and therefore we first describe the data set, and then we present the results in terms of reliability and delay. Sec. 5 concludes the paper.

80 2. Statement of the problem and related work

In this section, we first describe the scenario by detailing the assumed properties of the wireless communication system under study. In particular, we discuss the channel and the interference models, and we comment on the traffic model, and on the feedback. We then present some relevant results found in the recent literature, which are leveraged when defining the proposed allocation scheme. More specifically, the focus is on the tight upper bounds derived analytically for the average and maximal error probability for a codeword in the finite blocklength regime.

As a reminder, the main problem addressed in this work is the investigation of the most efficient redundancy allocation in the scenario considered. In this scenario, the wireless communication channel is described by a constant crossover probability over 
the duration of a codeword. However, as propagation delays are large relative to the correlation time of the channel fluctuations, the crossover probability can vary among different packets.

\subsection{Description of the scenario}

The considered scenario consists of a point-to-point wireless communication link between a transmitter and a receiver. The propagation delay, $\tau$, between the two is assumed to be much larger then the duration of a packet, $D_{p k t}$, e.g., $\tau=12.5 D_{p k t}$. This is typical in an underwater acoustic communication system, where packets $1 \mathrm{~kb}$ long at $6.25 \mathrm{kbps}$ lasts $160 \mathrm{~ms}$ and the propagation delay is $2 \mathrm{~s}$, e.g., in a link that is 3 $\mathrm{km}$ long with a nominal sound velocity of around $1500 \mathrm{~m} / \mathrm{s}$.

Both transmitter and receiver are assumed static, thus making the assumption of a slowly time-varying channel acceptable, as the communication channel only varies with the same rate of the changes in the surrounding environment. A slow time-varying quality of the communication means that the random process for bit errors is constant over the duration of a packet, but can change among different packets. In particular, the error process inside a packet is assumed to be binary, i.e., bits can be flipped, and symmetric, i.e., errors on $0 \mathrm{~s}$ and $1 \mathrm{~s}$ are equiprobable, and therefore it can be represented by one parameter, $\delta$, which is the bit error probability. Furthermore, bit errors are assumed to be independent of each other, which is reasonable in the presence of an interleaver that compensates for a possible memory in the error process due to inter-symbol interference.

Multiple-user interference is absent, as a medium access control has previously allocated the channel to the considered transmitter-receiver pair. This means that the communication performance depends solely on the channel quality. In fact, our main focus is on making it efficient to transmit as many bits as possible. As typical applications of underwater networks, where a network is defined as two or more nodes communicating, require very few nodes, we decided to first address the problem of a reliable and efficient communication in a point-to-point link, and leave that in a multiple-users scenario as a next step in our future work.

The traffic model consists of a backlogged queue at the transmitter side, i.e., a 
continuous flow of data keeps arriving to a queue with infinite capacity. The receiver is allowed to feed back both an ACK upon the correct decoding of a packet and a probing sequence used by the transmitter to monitor the communication performance of the link. As the communication system is half-duplex, this results in a packet delivery delay that lasts at least a round trip time, which is $2 \tau$, plus the duration of both the data packet and the probing sequence.

\subsection{Related work}

Recently, the authors of [16] have derived new tight upper bounds for the average and maximal error probability achievable with a given rate and blocklength. In the following, we limit the presentation to the results for the BSC, even though the scheme proposed in Sec. 3.1 is independent of the type of channel, as the only requirement is to have an analytical expression for the upper bound of the maximal error probability.

The codeword error probability is indicated with $\epsilon$ and depends on: $i$ ) the number of information bits, $x, i i)$ the number of redundancy bits, $y, i i i)$ the channel conditions, here for the BSC represented by $\delta \in] 0,1\left[\right.$ and $\delta \neq \frac{1}{2}$. We indicate with $n$ the blocklength of a codeword. The expression for the upper bound of the maximal error probability is derived from the computation of the achievable rate in the asymptotic regime once the blocklength, $n$, and the codeword error probability, $\epsilon$, are given.

The asymptotic behavior, i.e., for $n \rightarrow \infty$ and $\epsilon \rightarrow 0$, is also compared with the non-asymptotic upper and lower bounds derived in [17, Sec. III]. It turns out that the asymptotic expression is accurate even for short blocklengths and for rates much lower than the capacity, and for this reason we use the upper bound obtained in the asymptotic behavior. The achievable rate can be expressed as:

$$
\log M(n, \epsilon)=n C(\delta)-\sqrt{n \delta(1-\delta)} \log \left(\frac{1-\delta}{\delta}\right) Q^{-1}(\epsilon)+\frac{1}{2} \log n+O(1)
$$

$C(\delta)$ is the capacity of a BSC with crossover probability, $\delta$, i.e., $C(\delta)=1-h(\delta)$, where $h(\delta)$ is the entropy:

$$
h(\delta)=-\delta \log (\delta)-(1-\delta) \log (1-\delta) .
$$




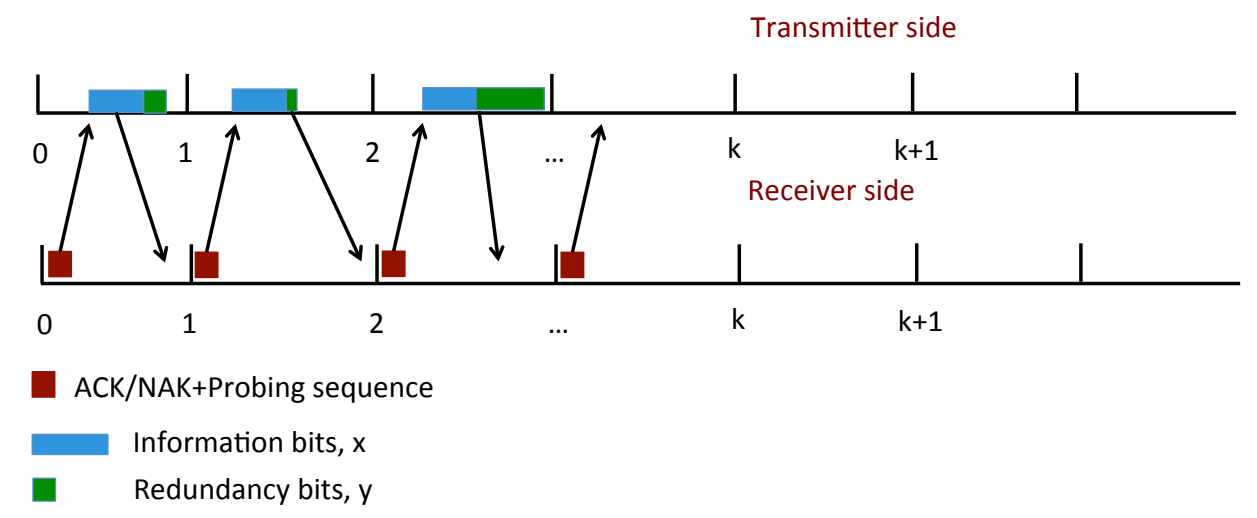

Figure 1: Representation of the communication system with the proposed redundancy allocation.

$Q^{-1}$ is the inverse of the standard Gaussian complementary cumulative distribution function. Therefore, the codeword maximal error probability, $\epsilon$, as a function of the number of information bits, $x$, the redundancy, $y$, and the crossover probability, $\delta$, can then be written as:

$$
\epsilon(x, y, \delta)=Q\left(\frac{(x+y) C(\delta)-x+\frac{1}{2} \log (x+y)}{\sqrt{(x+y) \delta(1-\delta) \log ^{2}\left(\frac{1-\delta}{\delta}\right)}}\right) .
$$

\section{Redundancy allocation techniques}

In this section, we describe the three allocation techniques that are then evaluated using experimental data in Sec. 4. These are an adaptive allocation, a constant redundancy allocation, and a rateless scheme.

\subsection{Proposed adaptive redundancy allocation}

The idea underlying the proposed allocation consists in adjusting the blocklength, $n$, depending on the current crossover probability, so that the resulting codeword is long enough to reliably deliver $x$ bits, but not too long so that bandwidth is not wasted. A representation is shown in Fig. 1. Packets consist of a constant blue portion, which contains the information bits, and of a green variable portion made of redundancy bits.

The duration of a time slot is Round Trip Time (RTT) $+D_{p k t}+D_{p r o b}$, where $D_{p k t}$ is 
the duration of the codeword and $D_{\text {prob }}$ is the duration of the probing sequence. Note that as $D_{p k t}$ is allowed to vary, so is the time slot duration.

This approach differs from using the achievable rate, as it indicates the maximum number of information bits that can be transmitted with a codeword $n$ bits long when a maximal codeword error probability, $\epsilon$, is tolerated. However, tracking the evolution of the codeword error probability, $\epsilon$, for time-varying channels could be costly in terms of number of packets that need to be transmitted, and inaccurate in the case of long propagation delays as experienced in underwater acoustic communications. For this reason, we would rather adjust the blocklength based on the crossover probability, $\delta$, which requires the transmission of only a probing sequence.

Therefore, we propose the following framework. We first define an 'efficiency' metric:

$$
\eta(x, y, \delta)=\frac{x(1-\epsilon(x, y, \delta))}{x+y},
$$

where $\epsilon(x, y, \delta)$ is the codeword error probability obtained in Eq. 3. $\eta$ represents the trade-off between reliability, indicated by the factor $1-\epsilon$, and spectral efficiency, expressed by the ratio $x /(x+y)$. Therefore, $\eta$ is a decreasing function of $y$, and an increasing function of $1-\epsilon$. However, $1-\epsilon$ itself is an increasing function of $y$, thus revealing a trade-off, which depends on how rapidly $\epsilon$ decreases as the redundancy increases. This trade-off gives rise to an optimum value $y_{o p t}$ that maximizes $\eta$. In other words, transmitting unnecessary bits for redundancy is a waste of power and bandwidth but providing insufficient redundancy results in a rise in packet failures and accompanying requests for retransmission which decreases efficiency.

The simple optimization problem is formalized as follows. Given the number of information bits $x$, and the bit error probability $\delta$, find the amount of redundancy, $y_{o p t}$ maximizing $\eta$, i.e.,:

$$
y_{\text {opt }}=\underset{y}{\operatorname{argmax}} \eta(x, \epsilon, y) .
$$

A maximizer is found by studying the sign of the first derivative of $\eta(x, y, \delta)$ with 


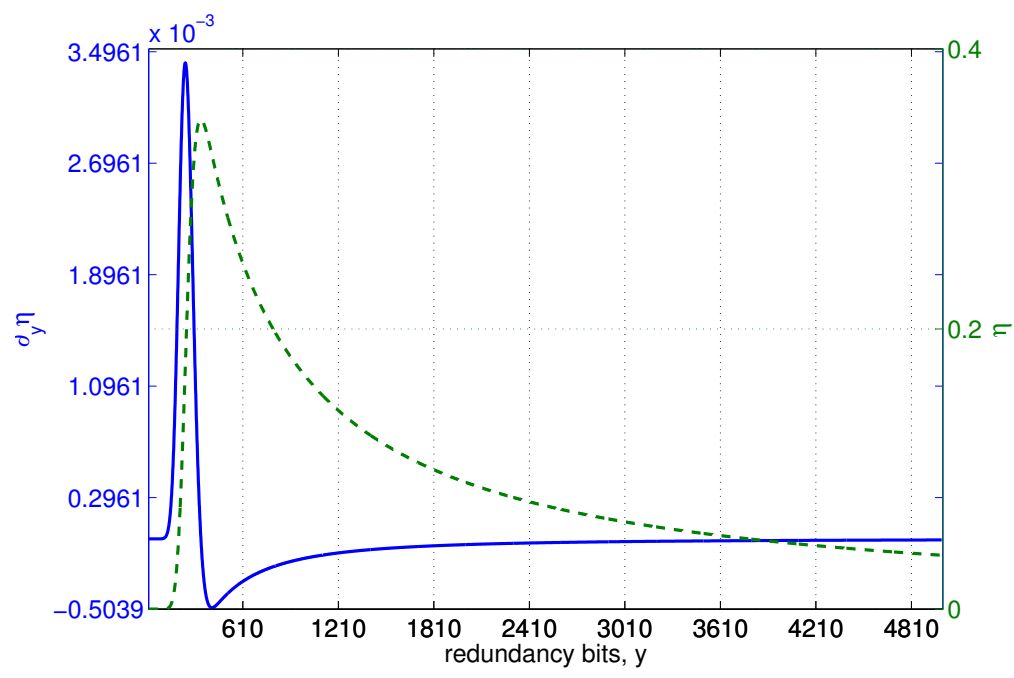

Figure 2: $\eta$ and its first derivative are represented by the green (dashed) and blue curves, respectively. In this case, $x=200$ bits and $\delta=0.046$.

respect to $y$, in symbols:

$$
\begin{aligned}
\frac{\partial \eta}{\partial y} & =\partial_{y}\left(\frac{x(1-\epsilon(x, y, \delta))}{x+y}\right) \\
& =\frac{-(x+y) x \partial_{y} \epsilon(x, y, \delta)-x(1-\epsilon(x, y, \delta))}{(x+y)^{2}}
\end{aligned}
$$

In the numerator, the first term has only positive factors, with the exception of $\partial_{y} \epsilon(x, y, \delta)$, which is negative, since $\epsilon$ is a strictly decreasing function of $y$. This makes the first term positive. Conversely, $x(1-\epsilon(x, y, \delta))$ is non-negative, since $x>0$ and $\epsilon(x, y, \delta) \in[0,1]$, thus making the second term non-positive. These considerations highlight that there exists a trade-off between the two terms, which determine the sign of $\partial_{y} \eta(x, y, \delta)$ as a function of $y$.

The derived maximizers are here shown in the following for values of $x$ lying in the interval $[200,1000]$, with an increment of 50 bits, and for values of $\delta$ spanning the interval $\left[10^{-3}, 0.1\right]$, with increments of 0.005 . In the basic case where no errors occurs, $\delta=0$, then no redundancy is allocated. In particular, Fig. 2 represents $\eta$ plotted with a dashed green line and its first derivative, plotted with a solid blue line, as a function of 


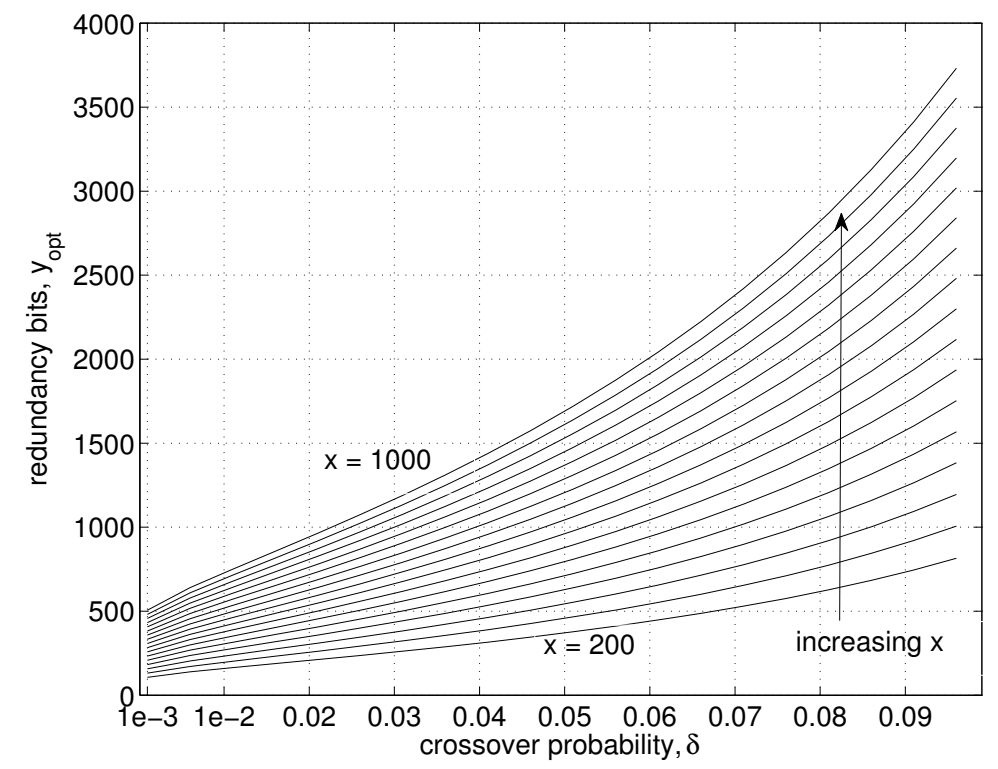

Figure 3: The $y$-axis represents the optimal number of redundancy bits, obtained from the optimization framework, for varying $x$ (represented by the different curves) and $\delta$ (in the $x$-axis).

$y$ for $x=200$ and $\delta=0.046$.

Fig. 3 shows the maximizers of $\eta$ as a function of $\delta$ in the aforementioned intervals. Each curve represents the optimal amount of redundancy for the considered values of $x$. In Sec.4 we will use the values of $y_{\text {opt }}$ obtained for $x=1000$, i.e., the upper curve.

Algorithms 1 and 2 summarize the proposed scheme at the transmitter and at the receiver, respectively. In few words, we assume that a lookup table of $\delta$ and $y_{\text {opt }}$ for the given $x$ is stored at the transmitter. Then, at the beginning of each slot, the receiver sends a probing sequence, from which $\hat{\delta}$ is estimated by the transmitter. The smallest $\delta$ larger than $\hat{\delta}, \delta^{\star}$, is then found in the lookup table, and the codeword to be transmitted will have blocklength equal to $x+y_{\text {opt }}\left(\delta^{\star}\right)$. If the bit error rate on the received codewords is not larger than $\delta^{\star}$, then the packet is successful.

In Sec. 4, we evaluate this scheme using experimental data and we also compare its performance with those obtained when a constant redundancy allocation and a rateless scheme are used. Therefore, for completeness we describe both these other schemes 

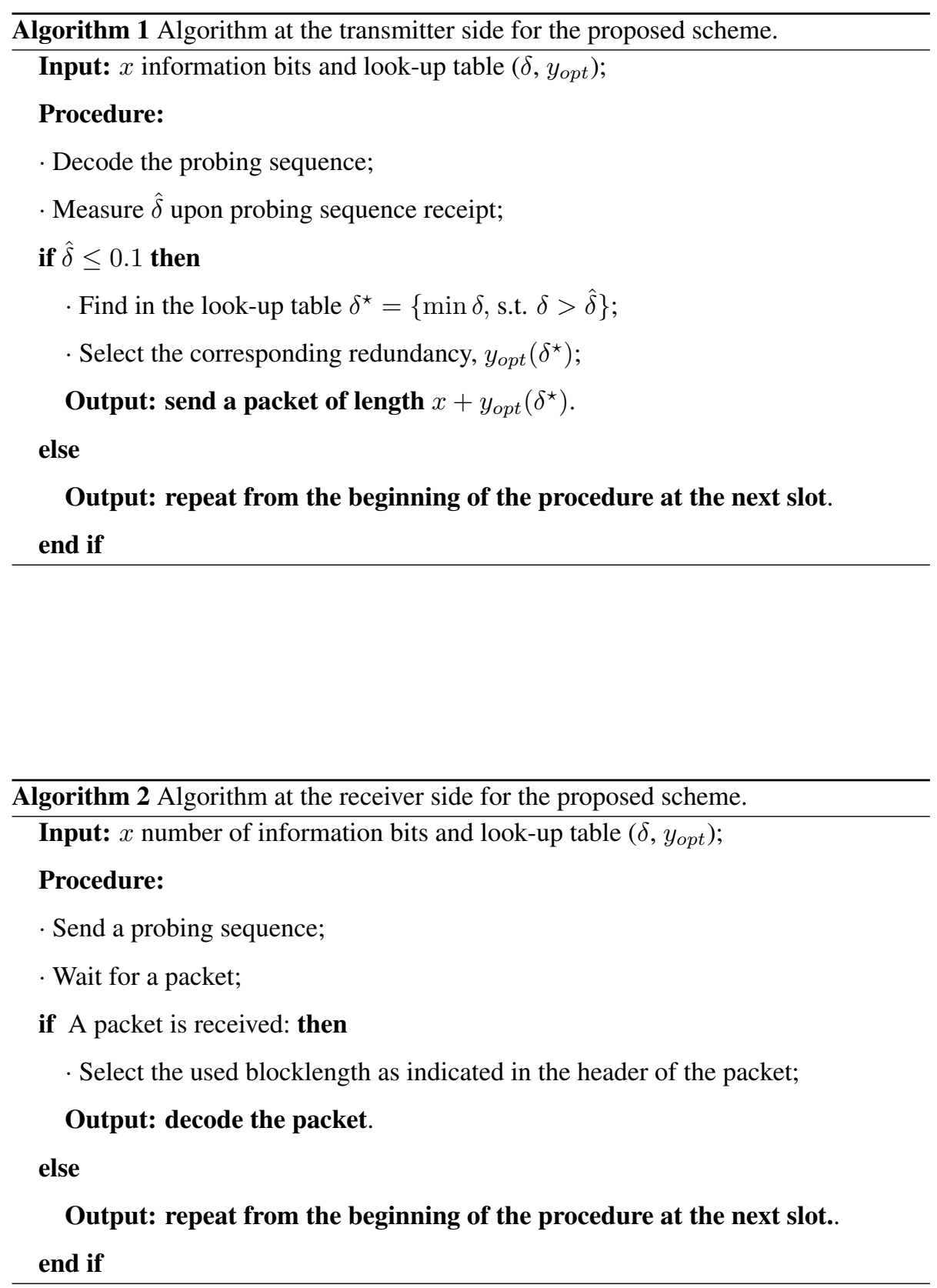


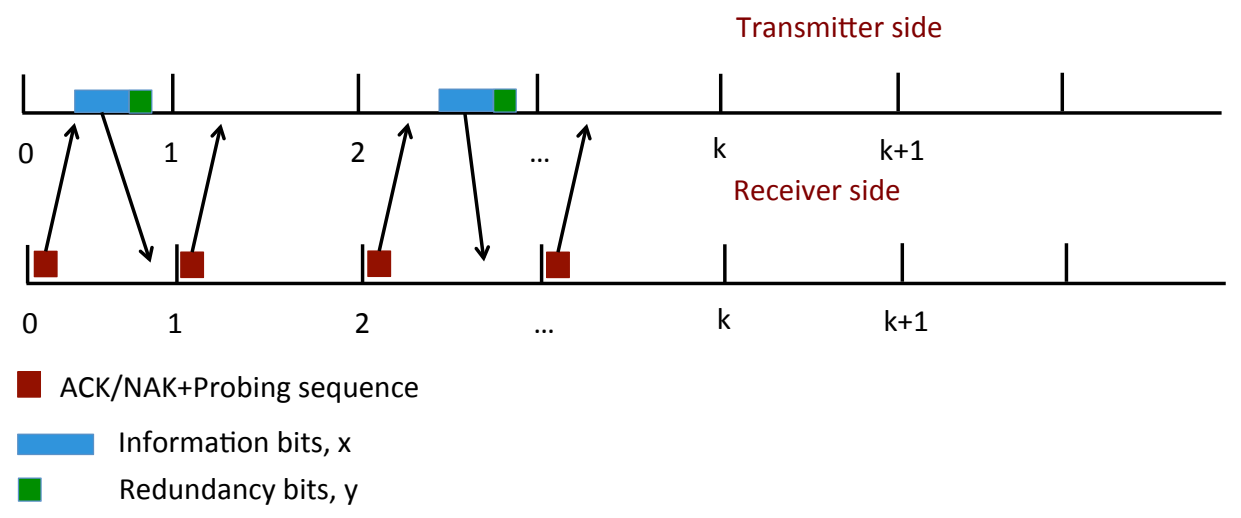

Figure 4: Representation of the communication system with constant redundancy allocation.

in the following by detailing the assumptions that we made in the implementation of these schemes.

\subsection{Constant redundancy allocation}

A pictorial representation of the constant redundancy allocation is in Fig. 4. The transmitter sends a codeword with a constant blocklength. Such constant blocklength is determined by using the maximizer of $\eta$ for a given bit error probability, $\delta_{i}$ and $x$, as described in Sec. 3.1. Therefore, whenever the bit error rate measured at the receiver, $\hat{\delta_{R x}}$, is larger than the $\delta_{i}$ used to compute the constant blocklength, the packet will fail. Therefore, a probing sequence, piggybacked to an ACK if a transmission was made in the previous slot, is sent at the beginning of the slot by the receiver. If the bit error rate measured at the transmitter, $\delta_{T x}$, is not larger than $\delta_{i}$, then a transmission is attempted, otherwise it is deferred until the next slot.

Also in Fig. 4 the blue portion of a packet contains information bits and the green part the constant number of redundancy bits. The red smaller packets sent by the receiver represent the probing sequence with a possible piggybacked ACK/NACK in case a transmission was attempted in the previous slot.

In Sec. 4, we consider two levels of robustness for the communication, i.e., when: a) redundancy is small, $y_{o p t}=507$, corresponding to $\delta_{i}=10^{-3}$, and $b$ ) redundancy is large $y_{\text {opt }}=3732$, corresponding to $\delta_{i}=0.1$. Therefore the blocklength for case $a$ ) is 


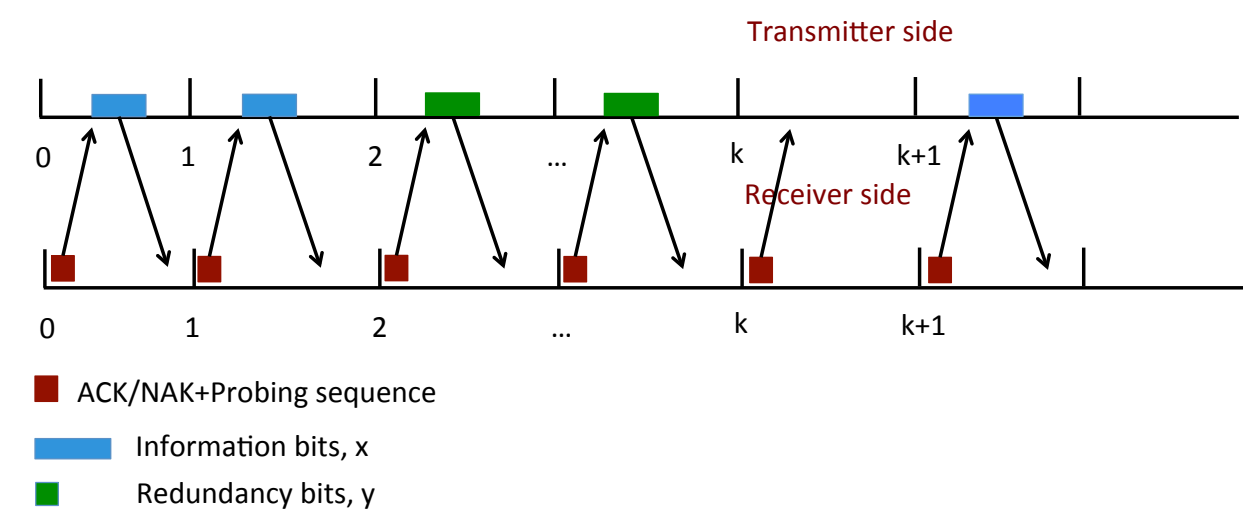

Figure 5: Representation of the communication system with a rateless coding scheme.

almost a third of that for case $b$ ), but it is also less robust. This comparison is meant to highlight the advantages and disadvantages of choosing low code rates in the context of underwater acoustic communications.

\subsection{Rateless scheme}

A rateless scheme consists in transmitting additional redundancy if the codeword made of the bits transmitted so far is not correctly received. As the amount of redundancy is adjusted on the fly and no rate is predefined, this scheme is called rateless. Its advantage over the constant allocation consists in transmitting as much redundancy as required to counteract the time-varying levels of reliability seen at each transmission.

A representation can be found in Fig. 5 First, all the information bits are transmitted, and then if they have not been correctly decoded by the receiver additional redundancy is transmitted. We assume that the packet length is equal to the smallest amount of redundancy derived in Sec. 3.1 i.e., 507 bits. For this reason, a single information frame, which is 1000 information bits long plus additional redundancy, requires the transmission of multiple packet 1 This choice of a short packet makes it possible to maintain a good resolution in adjusting the amount of redundancy, even though it taxes the delay with which a single information frame is delivered.

\footnotetext{
${ }^{1}$ In the performed simulations, the information bits consist of 2 packets.
} 
Every time a new packet is received, a decoding process starts anew with a codeword that consists of the bits sent up to the current slot juxtaposed with the additional redundancy received at the current slot. An information frame consists of at most 9 packets, which correspond to the longest blocklength used in the allocations described in Secs. 3.1 and 3.2. A probing sequence is sent in order to estimate the bit error rate and in case this is larger than $\delta_{\max }=0.1$ (which can be corrected with an information frame of 9 packets), then a transmission is deferred to the next slot.

Algorithms 3 and 4 summarize the procedures performed at the transmitter and receiver, respectively.

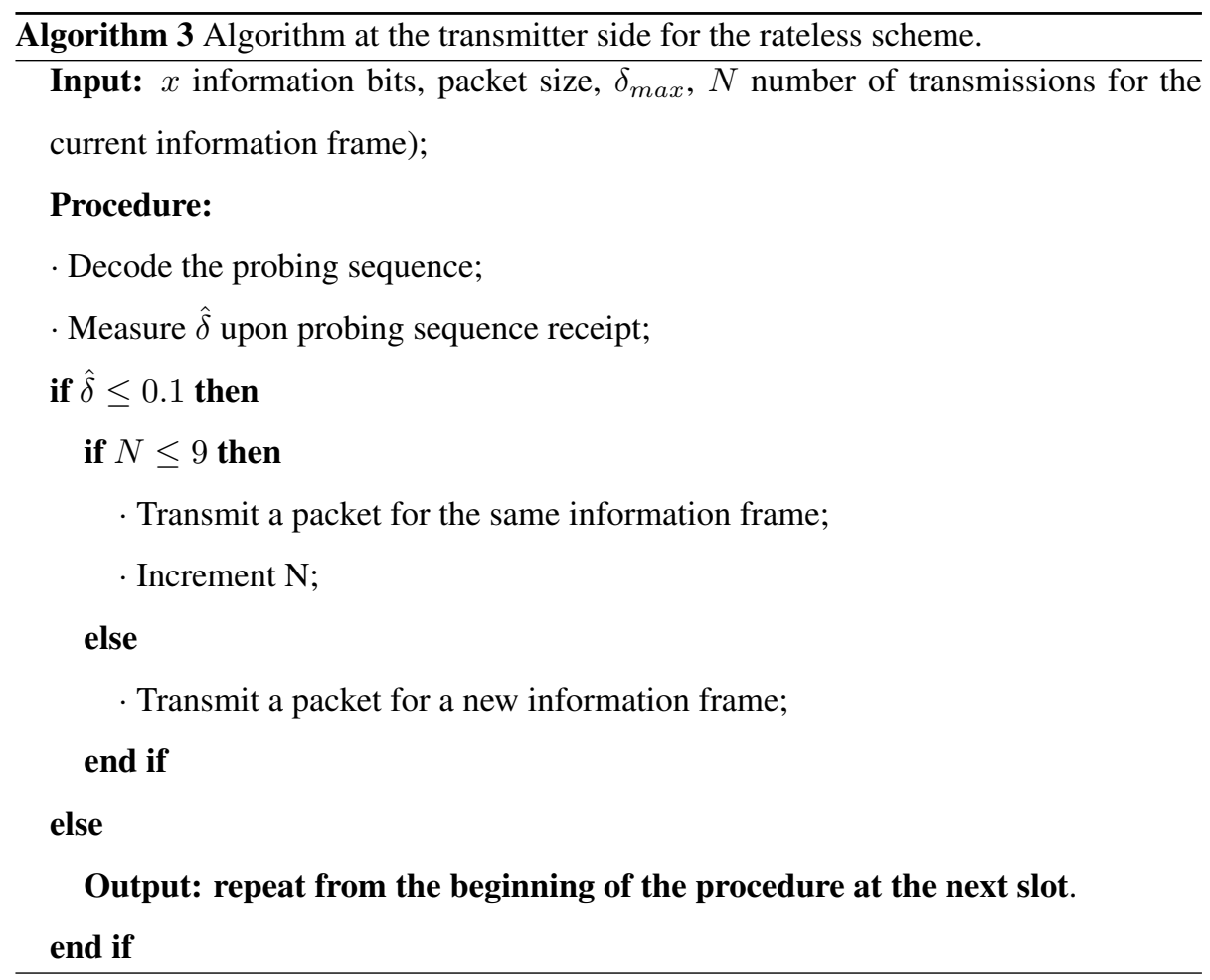

\section{Evaluation over experimental data}

In this section, we evaluate the described schemes using acoustic data collected at

sea. In Sec. 4.1, we provide some details on how the data set was collected and the 


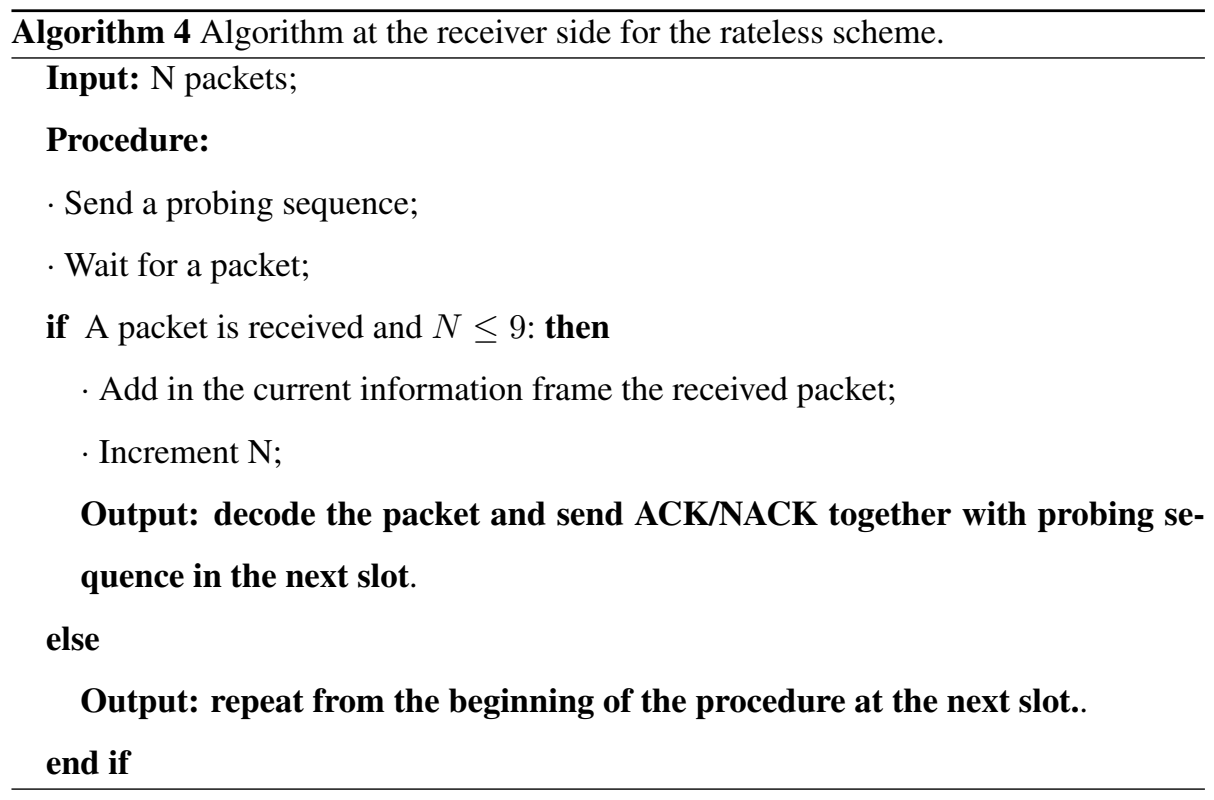

specifications of the communication system. Then in Secs. 4.2 and 4.3 we discuss the obtained performance in terms of reliability, efficiency, and delay.

\subsection{Description of data set}

The set of acoustic signals used in this paper were recorded during the KAM11 experimental trial [18]. Every two hours, bit streams made of nine one-minute long sequences were transmitted with a BPSK modulation, at center frequency $13 \mathrm{kHz}$ and rate 6250 bps. We consider the signals recorded at $3 \mathrm{~km}$ from the transmitter. Both the transmitter and the receiver were in the middle of the water column, i.e., at $45 \mathrm{~m}$ below the surface. The receiver consisted of an array of 24 hydrophones, of which we used 4 equally separated in space. The transmitter and the receiver were placed along an isobaric line. The results shown are relative to two Julian dates (JD), 187 at 4 am GMT and 188 at 10 am GMT, where the channel impulse responses were quite impulsive, as it can be observed from Figs. 6 and 7

Figs. 6 and 7 contain examples of the time series of the squared magnitude of the channel impulse response measured during the bit streams. The x-axis represents the delay referenced to some arbitrary zero delay point, with which different paths arrive 


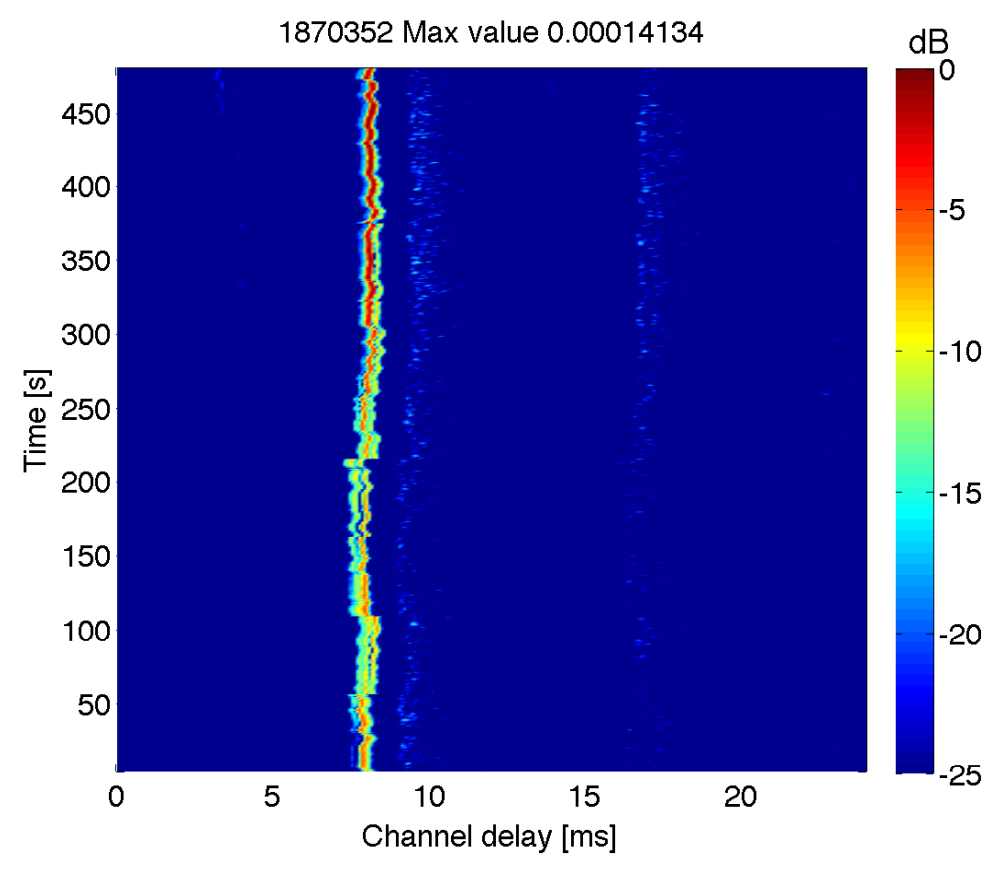

Figure 6: Time series of the squared magnitude (in $\mathrm{dB}$ with respect to the maximum value indicated) of the channel impulse response during Julian date 187 at GMT 03:52 am.

at the receiver (and it is not related to the propagation delay, $\tau=R T T / 2$ ). Since this stream of bits consists of trains of m-sequences, we estimated each channel impulse response by computing the auto-correlation of the 4095 bit long $\mathrm{m}$-sequence. The colormap of these figures corresponds to the value in $\mathrm{dB}$ relative to the maximum.

These two epochs are examples of when the channel impulse response contains only a few significant arrival, whose amplitude however varies significantly over time. Such variability is more likely to be caused by the focusing effect of surface waves. Since the arrival structure is quite concentrated over a main arrival, the channel estimation requires a small number of symbols. In fact, the computed results are obtained by setting the length of the feedforward filter of the Decision Feedback Equalizer (DFE derived in [19], to $0.5 \mathrm{~ms}$, and the length of the feedback filter to $1.5 \mathrm{~ms}$.

\footnotetext{
${ }^{2}$ The equalizer is used in training mode. It combines the signals measured at four channels spanning half a meter of the water column.
} 


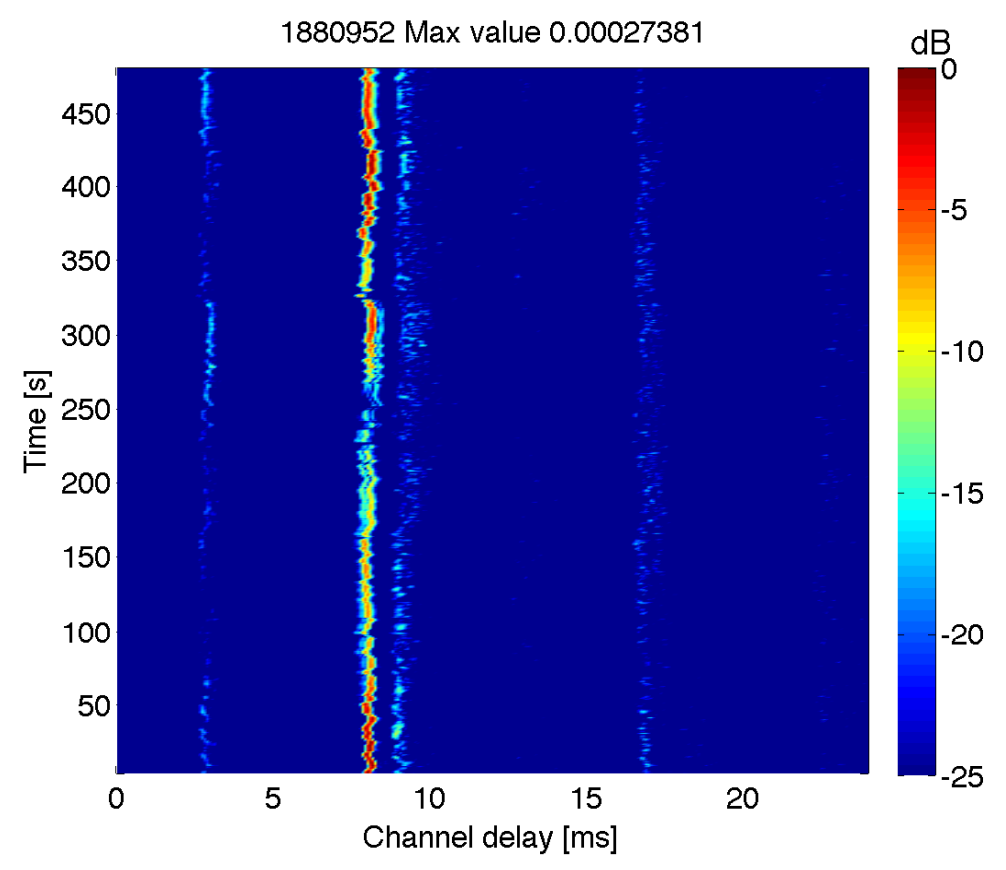

Figure 7: Time series of the squared magnitude (in $\mathrm{dB}$ with respect to the maximum value indicated) of the channel impulse response during Julian date 188 at GMT 09:52 am.

The time series of bit error rates computed over the 4095 bit long sequences is shown in Fig. 8, where the black markers refer to JD 187 and the red ones to JD 188. The $\mathrm{y}$-axis represents the time in seconds whereas the $\mathrm{x}$-axis represents the bit error rate (or crossover probability $\delta$ ). It can be noticed how the bit error rate drops from second 250 on in the black time series, i.e., as soon as the intensity of the main arrival increases as shown in Fig. 6 Similarly, during JD 188, the bit error rate increases when the main arrival fades around second 250.

In light of these observations, we now evaluate the three systems described earlier using these time series. These bit streams are therefore downsampled in order to reproduce the same evolution in time, i.e., accounting for the proper RTT that would be experienced at sea. This also means that as each scheme has different packet lengths, it can happen that transmissions occur at different times, and therefore at different channel conditions. Even though comparing the performance obtained by this study 


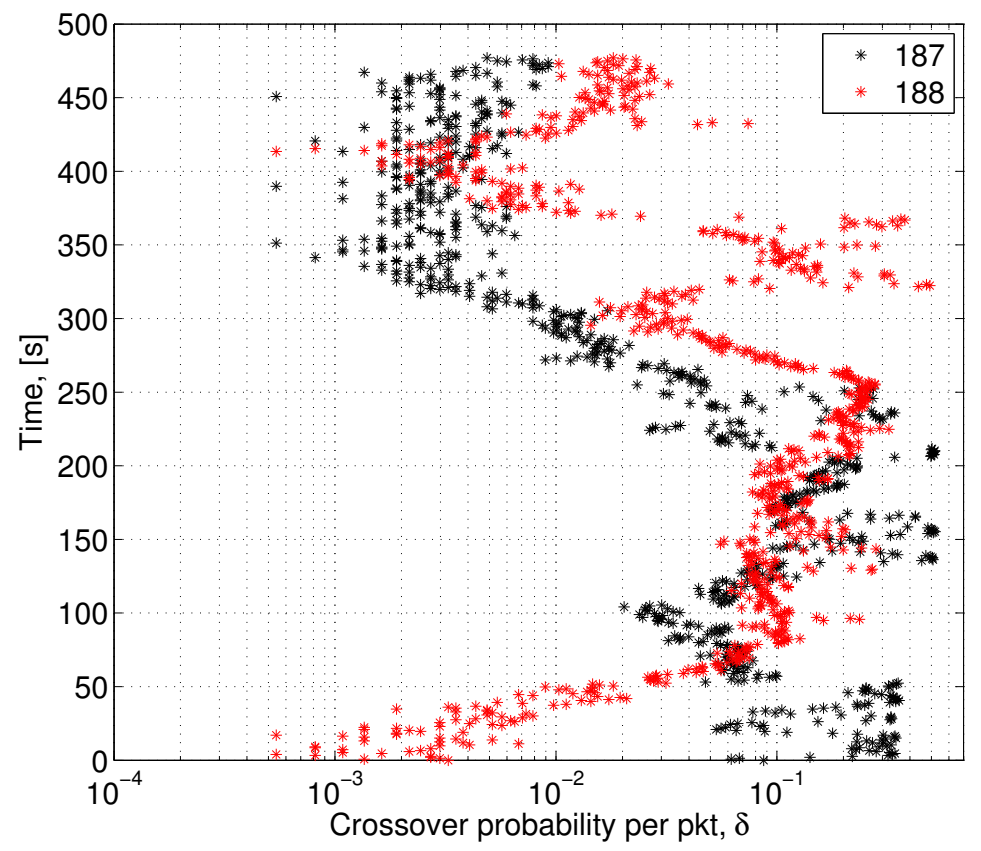

Figure 8: Time series of the bit error rate (or crossover probability) per packet estimated over packets 4095 bits long. Black and red markers correspond to JD 187 and 188, respectively.

might not be completely fair, as the three systems can downsample the time-varying error process at different times, it however helps in understanding the evolution and the behavior of the performance that would occur in a real scenario.

To better understand this, we show in Fig. 9 the time series of the SNR at the output of the equalizer, estimated over the packets that were successfully transmitted for each scheme. Blue and red markers refer to the constant redundancy allocation with a small and large robustness, respectively. As the blue markers correspond to less robust transmissions, they basically occur only at high SNR (in fact no blue markers can be found between 60 and $250 \mathrm{~s}$ ), whereas more robust codewors can better cope with deep fades, as those around 60 and $250 \mathrm{~s}$ for both epochs. The black markers represent the SNR estimated over the packets with variable robustness, as shown by whether these markers are higher or lower than the red ones for some periods of time. This effect is caused by the slightly different sampling time of the time series, and by the 


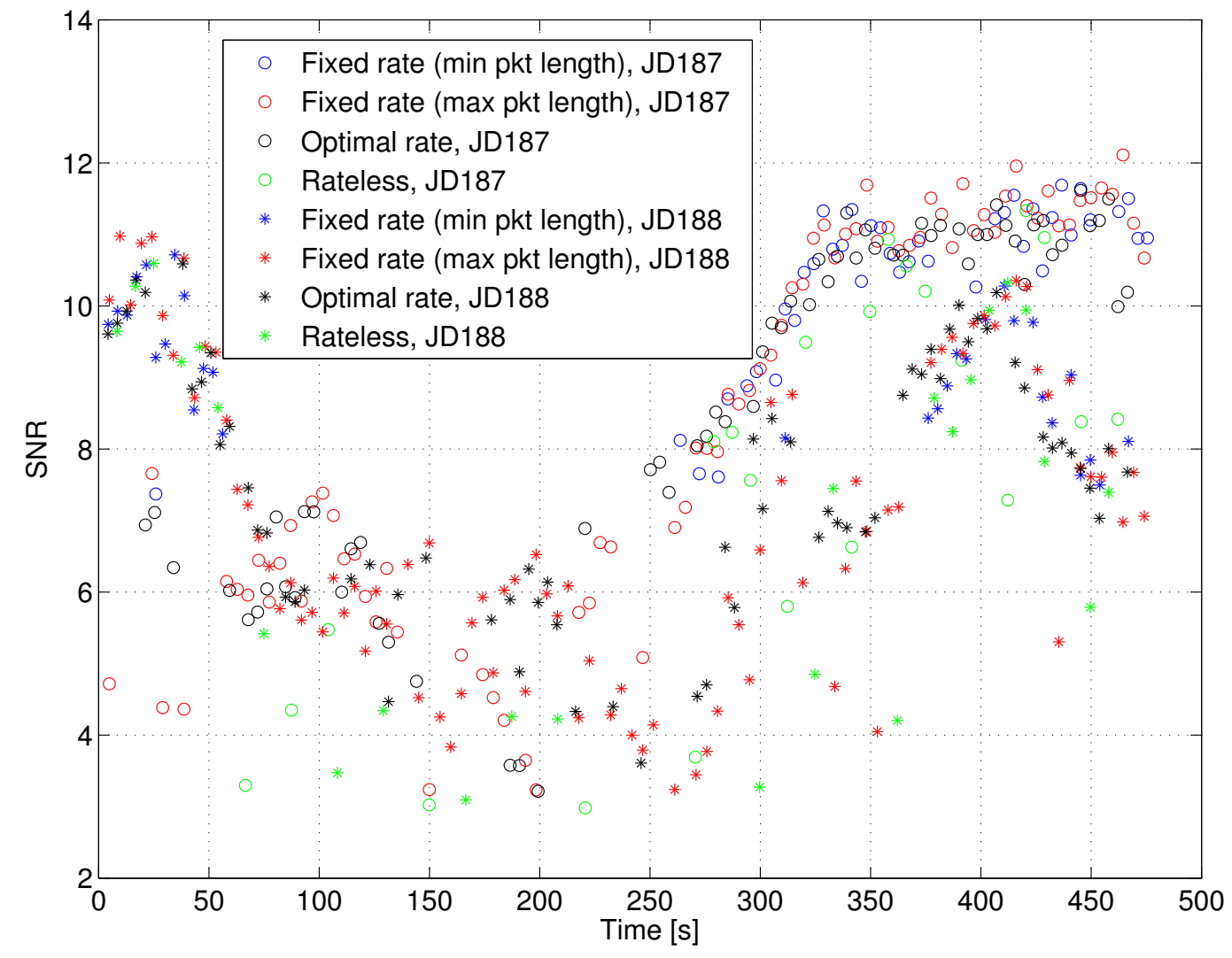

Figure 9: Time series of the SNR measured at successful transmissions made with the discussed allocation schemes. Circles and stars correspond to JD 187 and 188, respectively.

fact that the estimate of the SNR at the output of the equalizer decreases as the channel

impulse response varies, which is more likely during larger packets. This effect is even stronger for the SNR estimated over the information frames of the rateless scheme. In fact, when the SNR is low, more redundant transmissions are requested. However, these packets are separated in time by at least $4 \mathrm{~s}$ (which is the RTT). This means that the assembled information frame contains more diversity in the channel conditions, and the DFE performs worse, thus giving rise to lower SNR. 


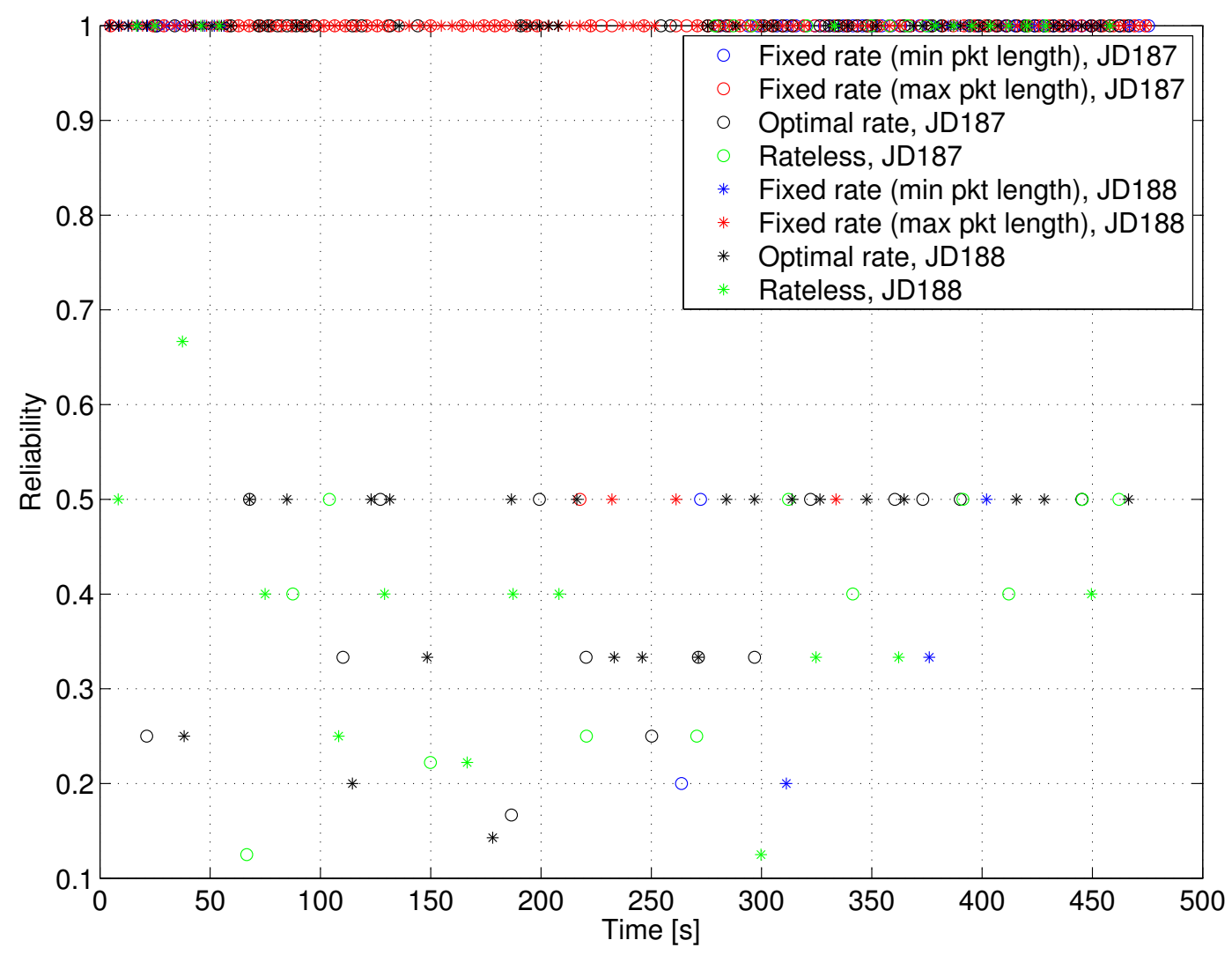

Figure 10: Time series of the reliability per delivered packet for the allocation schemes.

\subsection{Efficiency}

In this section, we analyze the performance in terms of reliability, effective transmission rate, and efficiency, per successful packet. In particular, the reliability is computed as the inverse of the number of transmission attempts made before the successful delivery of $x$ bits of information. In other words, retransmissions are performed until the packet is delivered successfully. Fig. 10 shows the time series of the reliability for 
the different schemes. When a transmission is attempted ${ }^{3}$ and successful at the first shot then the reliability is 1 . This happens for the majority of the transmissions made by the scheme with constant reliability and large redundancy. Since the CSI estimated at the transmitter can become outdated, some retransmissions are also requested in the proposed scheme, thus reducing the reliability. For the rateless scheme, the reliability is computed as 2 over the number of transmissions attempts. In fact, in order to transmit 1000 bits, the transmitter needs to send at least 2 packets. For this scheme many more attempts per successful packet are performed due to the lower SNR experienced by the information frames, as previously explained with Fig. 9 .

The efficiency per delivered packet is then computed as the product of the reliability and the ratio between $x$ and the overall number of bits transmitted until the delivery of the packet. Its time series is shown in Fig. 11 for the different allocation schemes. The constant rate allocations have mostly a constant efficiency equal to the rate, as the first transmission attempt is also successful in the most cases. Instead, the proposed scheme presents a higher efficiency on average, except in those cases where outdated estimates of the bit error rate mislead the rate selection process and more transmissions have to be performed. The rateless scheme is on average much less efficient than the others as it requires many transmissions. Therefore, if reliability is the most important metric, then a robust fixed rate allocation would be the right choice for underwater acoustic communications. However, if also efficiency is important, i.e., if the amount of energy put in the water should also be minimized, then the proposed scheme outperforms the others at the cost of a slightly lower reliability, caused by outdated estimate of the crossover probability.

Fig. 12 represents the actual transmission rate for each value of the reliability per successful packet. The actual transmission rate is the ratio between $x$ and the overall number of bits transmitted until the packet is correctly decoded. This figure is useful to understand whether the decrease of the actual rate is due to the loss of packets or to the allocation of more redundancy. The reliability of a constant redundancy alloca-

\footnotetext{
${ }^{3}$ We recall that if the $\hat{\delta}$ estimated over the probing sequence is too large then a transmission is deferred, as mostly happens for short packets (blue markers) between $50 \mathrm{~s}$ and $250 \mathrm{~s}$.
} 


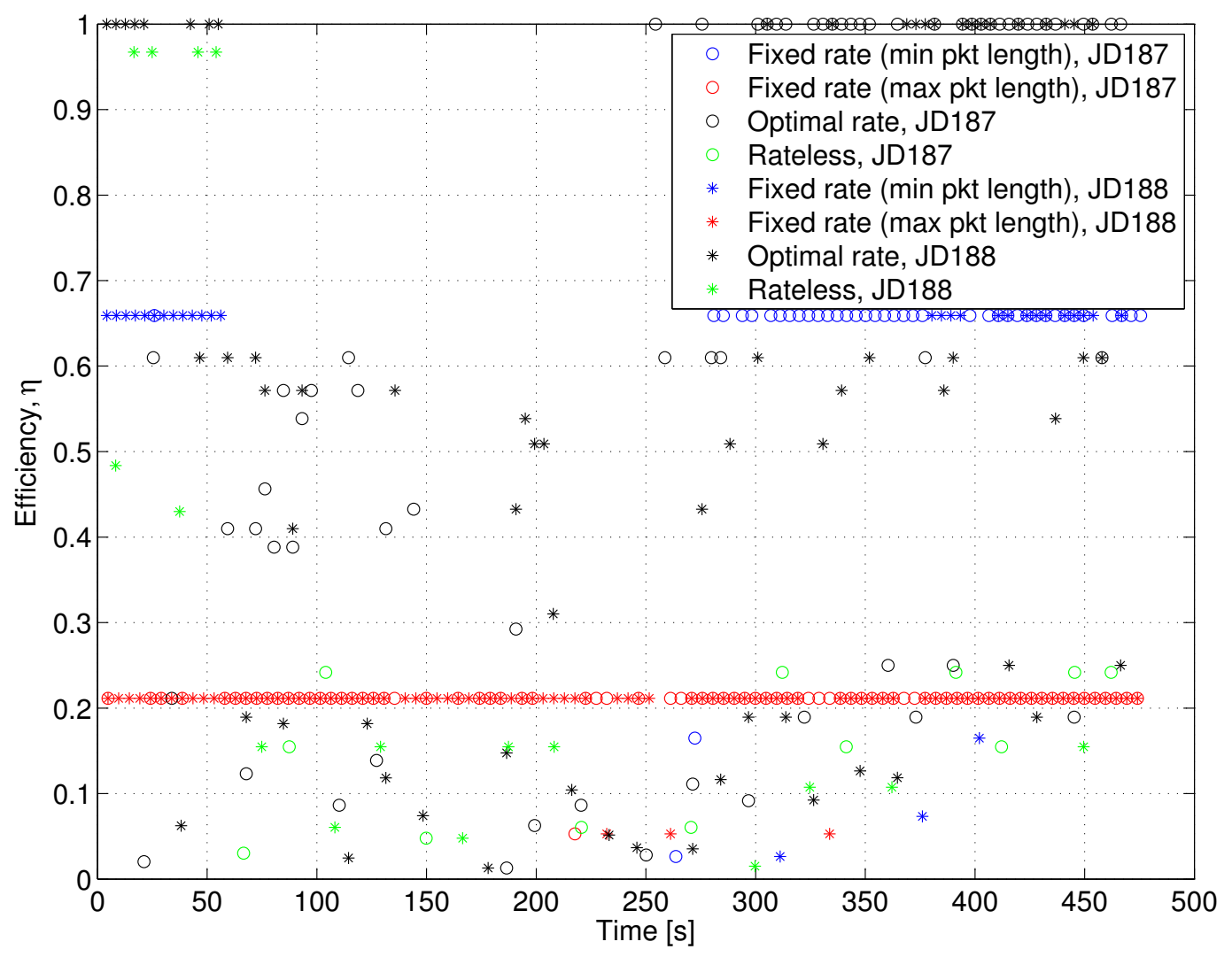

Figure 11: Time series of the efficiency per successful packet for the analyzed schemes. most of the realizations fall in the pair $(1, x / n)$ for both blue and red markers. The two other blue markers and the red marker corresponding to a smaller reliability are seldom obtained when the transmission attempt fails. For the proposed scheme, the multiple values of rate for reliability 1 indicate that the system is actually adapting to the channel conditions in an efficient way. Most of the points are in this area of the plot. Similarly, the several values of the rate for a given level of reliability indicates that even though the system adapts to the channel conditions, it overestimates the quality of the communication performance by relying on an outdated information. The smaller the 


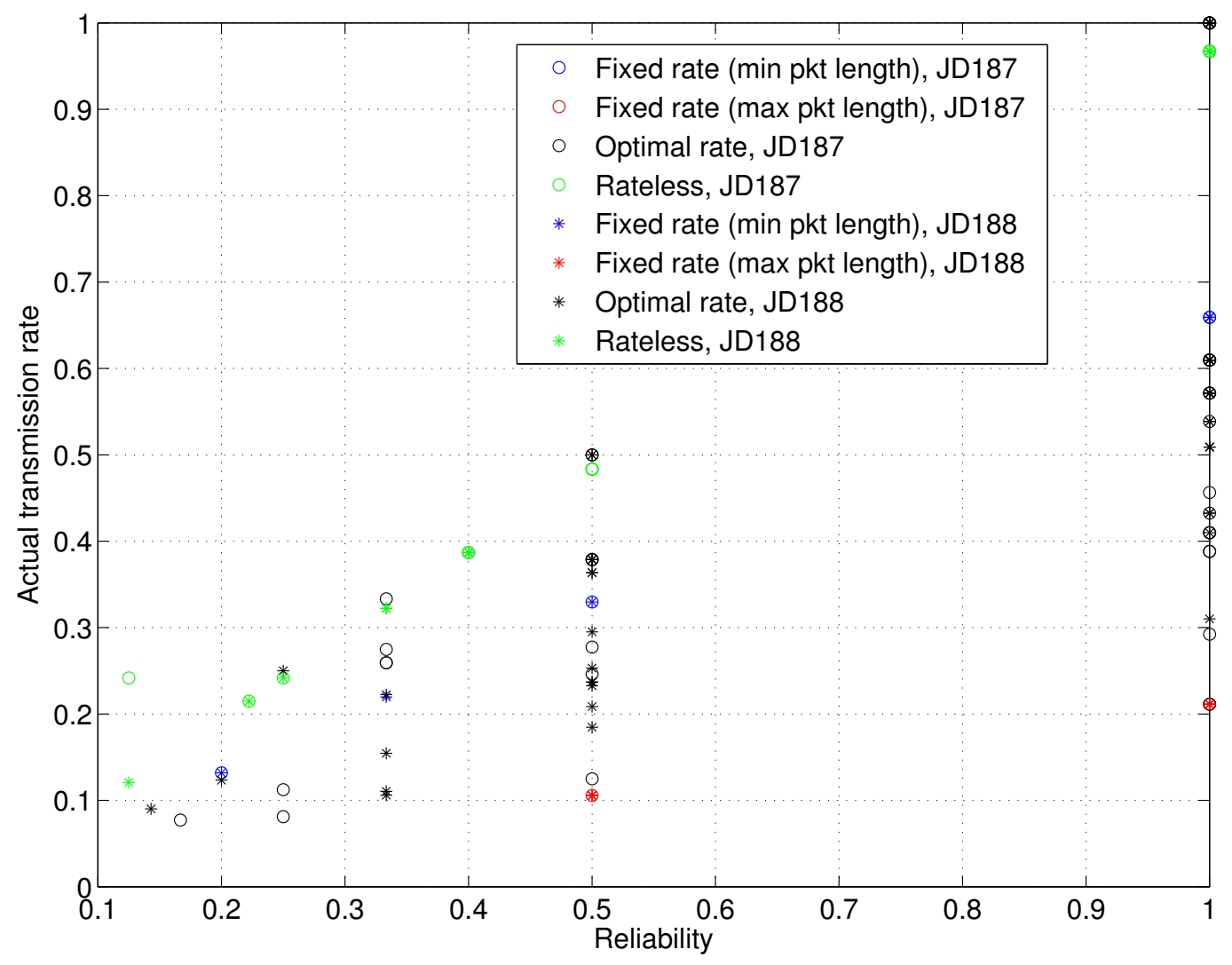

Figure 12: Actual transmission rate vs reliability per successful packet for the considered schemes.

reliability (which implies a moment of low SNR), the fewer the options in selecting the rate, which implies that the vertical lines collapse to one point. The rate obtained with the rateless scheme is linearly dependent on the reliability, since an information frame fails due to a low SNR at the output of the equalizer. Such decrease of SNR is due to the fact that packets with different channel conditions (at least $4 \mathrm{~s}$ apart) are juxtaposed, thus taxing the performance of the equalizer. This effect remains until enough redundancy is transmitted to balance this loss of communication performance due to the channel variability. 


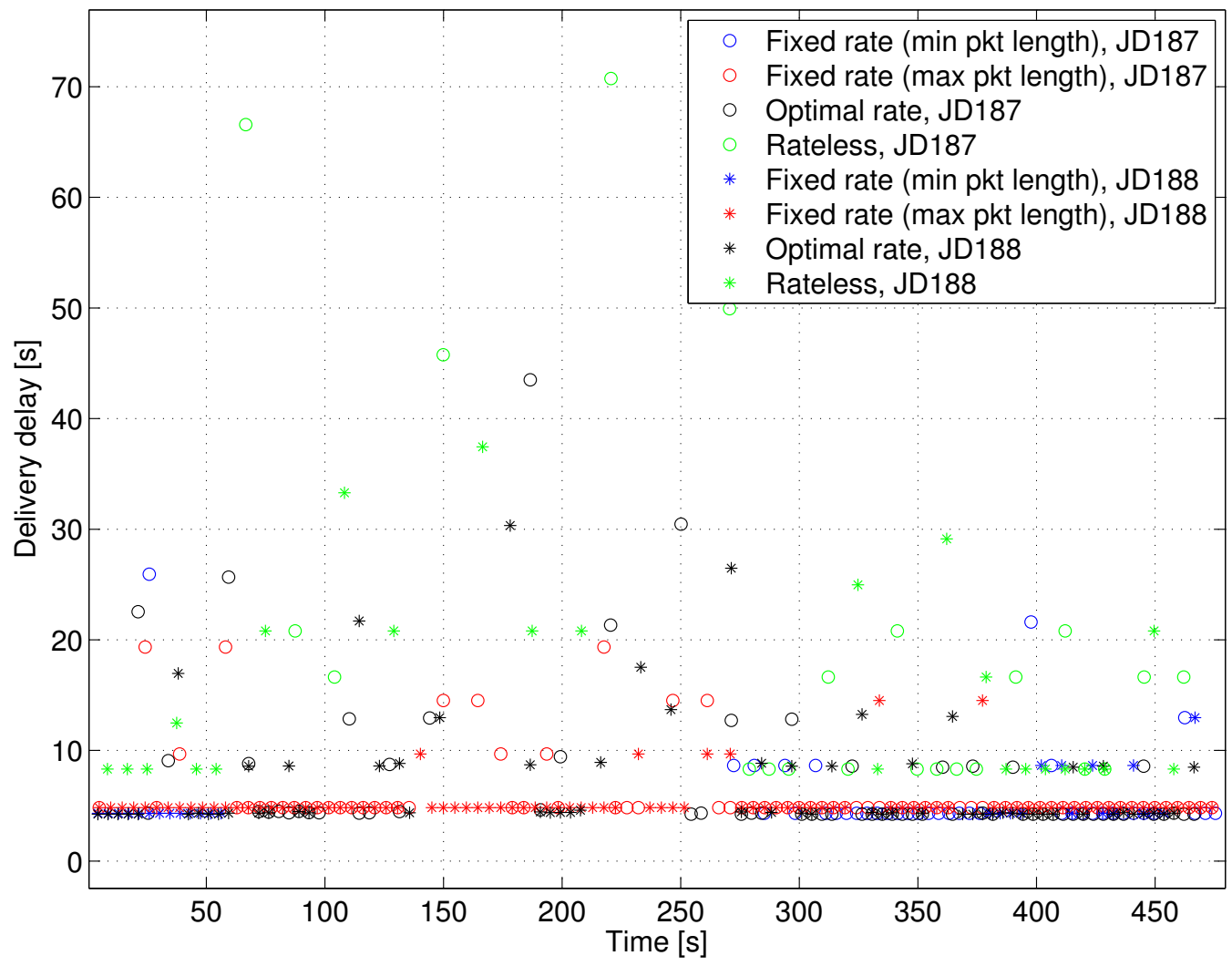

Figure 13: Time series of the packet delivery delay per successful packet for the analyzed allocation schemes.

\subsection{Packet delivery delay}

Another important metric is the time measured between two successful consecutive packets, i.e., the delivery delay. Fig. 13 shows the time series of this delay per successful packet for the different allocation schemes. The fixed allocation with large redundancy, indicated with red markers, has small delay as the first attempt is also successful. The proposed scheme has slightly lower delays when the first attempt is successful, but since the RTT (4 s) is much larger than the packet length, having adaptive blocklengths is not much advantageous in terms of delay. The rateless scheme has

the largest delay as 2 packets need to be transmitted just for the information bits. 


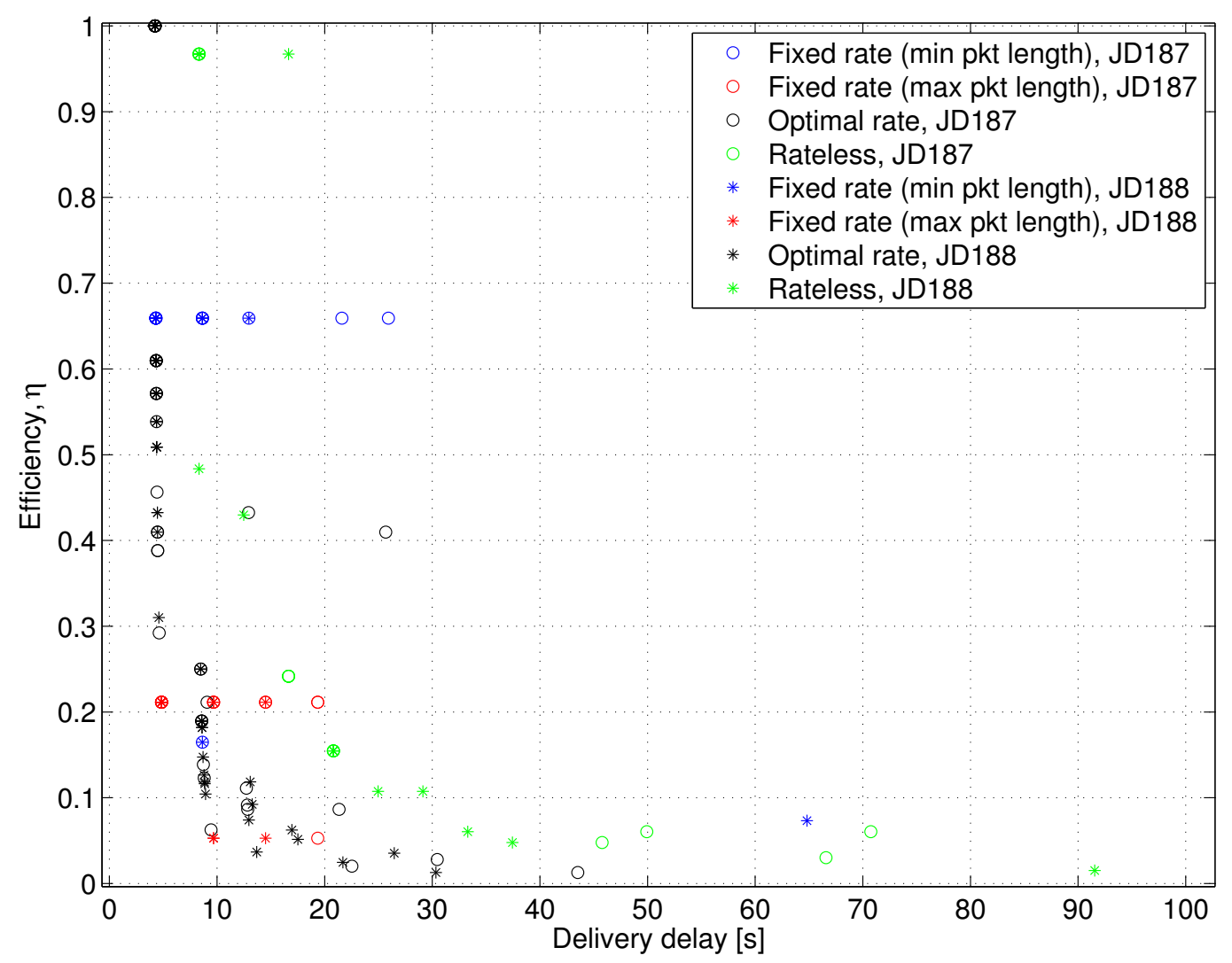

Figure 14: Efficiency vs delay for the analyzed allocation schemes

Fig. 14 represents the results for the measured efficiency given the delivery delay for all schemes. Note that each point represents different occurrences of the specific ( $\eta$,delay) pair, and for this reason, this plot should be interpreted by also referring to the previously presented time series in Figs. 13 and 11 . In particular, the red and blue markers corresponding to small values of delays are more frequent as shown in the time series, and therefore more representative. From this figure, we can conclude that the proposed scheme has higher efficiency than the other schemes without affecting the delivery delay. The considered rateless scheme indicated with green markers has instead the worst performance in terms of delay, but when the channel conditions are 
favorable, it can be more efficient than a constant rate allocation scheme.

\section{Conclusions}

In this paper, we have proposed a redundancy allocation that leverages recent results on the maximal error probability for fixed rate and finite blocklength. We have then evaluated the performance of the proposed scheme in terms of reliability, efficiency, and delay. Such evaluation was performed over experimental data collected over a link $3 \mathrm{~km}$ long.

The performance of the proposed scheme has been compared with those obtained for a constant rate allocation and a rateless scheme. Results confirm that in the presence of a time-varying channel with long propagation delays, the utilization of a robust codeword is inefficient in terms of used bandwidth and energy per bit. However, as it results in fewer retransmissions, it also guarantees short delivery delays. Therefore, in the case where a lower reliability can be tolerated, the proposed scheme provides higher efficiency and shorter delivery delays.

\section{Acknowledgment}

This work was supported by ONR under Grants no. N00014-13-10142, N0001407-10738 and N00014-11-10426, and by the European Commission under the Seventh Framework Programme (Grant Agreement 258359 - CLAM).

\section{References}

[1] WHOI umodem.

[2] Evologics acoustic modems.

URL http://www.evologics.de/en/products/acoustics/ index.html 
[3] E. Uysal-Biyikoglu, B. Prabhakar, A. El Gamal, Energy-efficient packet transmission over a wireless link, IEEE/ACM Transactions on Networking 10 (4) (2002) 487-499.

[4] E. Uysal-Biyikoglu, A. El Gamal, On adaptive transmission for energy efficiency in wireless data networks, IEEE Transactions on Information Theory 50 (12) (2004) 3081-3094.

[5] M. Zafer, E. Modiano, Optimal rate control for delay-constrained data transmission over a wireless channel, IEEE Transactions on Information Theory 54 (9) (2008) 4020-4039.

[6] J. Lee, N. Jindal, Energy-efficient scheduling of delay constrained traffic over fading channels, IEEE Transactions on Wireless Communications 8 (4) (2009) 1866-1875.

[7] R. Srivastava, C. Koksal, Energy optimal transmission scheduling in wireless sensor networks, IEEE Transactions on Wireless Communications 9 (5) (2010) $1550-1560$.

[8] R. Berry, R. Gallager, Communication over fading channels with delay constraints, IEEE Transactions on Information Theory, 48 (5) (2002) 1135-1149.

[9] P. Casari and M. Rossi and M. Zorzi, Towards optimal broadcasting policies for HARQ based on fountain codes in underwater networks, in: Proc. IEEE/IFIP WONS, 2008.

[10] P. Casari and A. F. Harris III, Energy-efficient reliable broadcast in underwater acoustic networks, in: Proc. ACM WUWNet, 2007.

[11] W. Zhang, U. Mitra, A delay-reliability analysis for multihop underwater acoustic communication, in: Proc. ACM WUWNet, 2007, pp. 57-64.

[12] H. Zhuang, H. Tan, A. Valera, Z. Bai, Opportunistic ARQ with bidirectional overhearing for reliable multihop underwater networking, in: Proc. IEEE OCEANS, 2010, pp. 1-6. 
[13] F. Qu, L. Yang, Rate and reliability oriented underwater acoustic communication schemes, in: Proc. IEEE DSP/SPE, 2009, pp. 144-149.

[14] U. Erez, G. Wornell, A super-Nyquist architecture for reliable underwater acoustic communication, in: Proc. Communication, Control, and Computing (Allerton), 2011, pp. 469-476.

[15] N. Bonello, R. Zhang, S. Chen, L. Hanzo, Reconfigurable rateless codes, IEEE Transactions on Wireless Communications, 8 (11) (2009) 5592-5600.

[16] Y. Polyanskiy, Channel coding: non-asymptotic fundamental limits, Ph.D. dissertation, Princeton Univ., Princeton, NJ, USA, 2010.

[17] Y. Polyanskiy, H. Poor, S. Verdu, Channel coding rate in the finite blocklength regime, IEEE Transactions on Information Theory 56 (5) (2010) 2307-2359.

[18] P. L. Nielsen, M. Siderius, F. B. Jensen, Kauai Acomms MURI 2011 (KAM11)Experiment, in: Proc. of the European Conf. on Underwater Acoustics, 2012.

[19] J. C. Preisig, Performance analysis of adaptive equalization for coherent acoustic communications in the time-varying ocean environment, The Journal of the Acoustical Society of America 118 (1) (2005) 263-278. 
Beatrice Tomasi received the $\mathrm{PhD}$ in Information Engineering in 2011 at the University of Padova, Italy, where she was a postdoctoral research fellow during 2012. She has been actively working on underwater acoustic channel modeling and the performance evaluation of communication systems and networking protocols for underwater acoustic networks. She was a visiting student at the NATO Undersea Research Center (NURC, today CMRE) during 2009 and at the Woods Hole Oceanographic Institution (WHOI) in 2011, where she is currently a postdoctoral research fellow. She actively participated in the Italian project NAUTILUS, and was involved in research efforts funded by the European Community. Her research interests include energy efficient protocol for underwater acoustic networks and their impact on marine mammals.

Daniele Munaretto received the BS and MS degrees (with honors) in Telecommunications Engineering in 2004 and 2007, respectively, at the University of Padova, Italy. After an internship for his MS thesis at DOCOMO Euro-Labs, in Munich, Germany (2006-2007), he joined EuroLabs from 2007 to 2010 as R\&D Engineer in the Ubiquitous Networking Research Group. He is currently about to achieve the Ph.D. title at the Department of Information Engineering at the University of Padova, under the supervision of Prof. M. Zorzi. His research interests mainly cover resource allocation in wireless networks, network coding, video streaming services and P2P networks. He authored several scientific papers and EU, US, and JP patents.

Dr. Preisig received the B.S. degree in electrical engineering from the United States Coast Guard Academy in 1980 and served on active duty in the Coast Guard until August 1985. In 1985, he entered the Massachusetts Institute of Technology as a graduate student and received the S.M. and E.E. degrees in electrical engineering from the Massachusetts Institute of Technology in 1988, and the Ph.D. degree in electrical and ocean engineering from the Massachusetts Institute of Technology/Woods Hole Oceanographic Institution Joint Program in Oceanography and Oceanographic Engineering in 1992. He was a Postdoctoral Investigator at WHOI from 1992 to 1994 and a Visiting Assistant Professor at Northeastern University from 1994 to 1997. Since July 1997, he has been on the scientific staff of the Department of Applied Ocean Physics and Engineering at WHOI and is currently an Associate Scientist with Tenure.

He is the recipient of the 1999 ONR Ocean Acoustics Young Faculty Award and is a Fellow of the Acoustical Society of America. He is a member of the ASA's Underwater Acoustics and Signal Processing Technical Committees. Dr. Preisig is also an associate editor of the IEEE Journal of Oceanic Engineering and served as a member of the IEEE Sensor Array and Multichannel Signal Processing Technical Committee from 1998 to 2004. Dr. Preisig's research interests are in the areas of adaptive signal processing, system identification, underwater acoustic propagation modeling, underwater acoustic communications, and numerical optimization.

Michele Zorzi received his Laurea and $\mathrm{PhD}$ degrees in electrical engineering from the University of Padova in 1990 and 1994, respectively. During academic year 1992-1993 he was on leave at UCSD, attending graduate courses and doing research on multiple access in mobile radio networks. In 1993 he joined the faculty of the Dipartimento di Elettronica e Informazione, Politecnico di Milano, Italy. After spending three years with the Center for Wireless Communications at UCSD, in 1998 he joined the School of Engineering of the University of Ferrara, Italy, where he became a professor in 2000. Since November 2003 he has been on the faculty of the Information Engineering Department at the University of Padova. His present research interests include performance evaluation in mobile communications systems, random access in mobile radio networks, ad hoc and sensor networks, energy constrained communications protocols, and underwater communications and networking.

He was Editor-In-Chief of IEEE Wireless Communications from 2003 to 2005 and Editor-InChief of the IEEE Transactions on Communications from 2008 to 2011, and serves on the Editorial Board of the Wiley Journal of Wireless Communications and Mobile Computing. He 
was also guest editor for special issues in IEEE Personal Communications ("Energy Management in Personal Communications Systems") and IEEE Journal on Selected Areas in Communications ("Multimedia Network Radios" and "Underwater Wireless Communications and Networking"). He served as a Member-at-Large of the Board of Governors of the IEEE Communications Society from 2009 to 2011. 


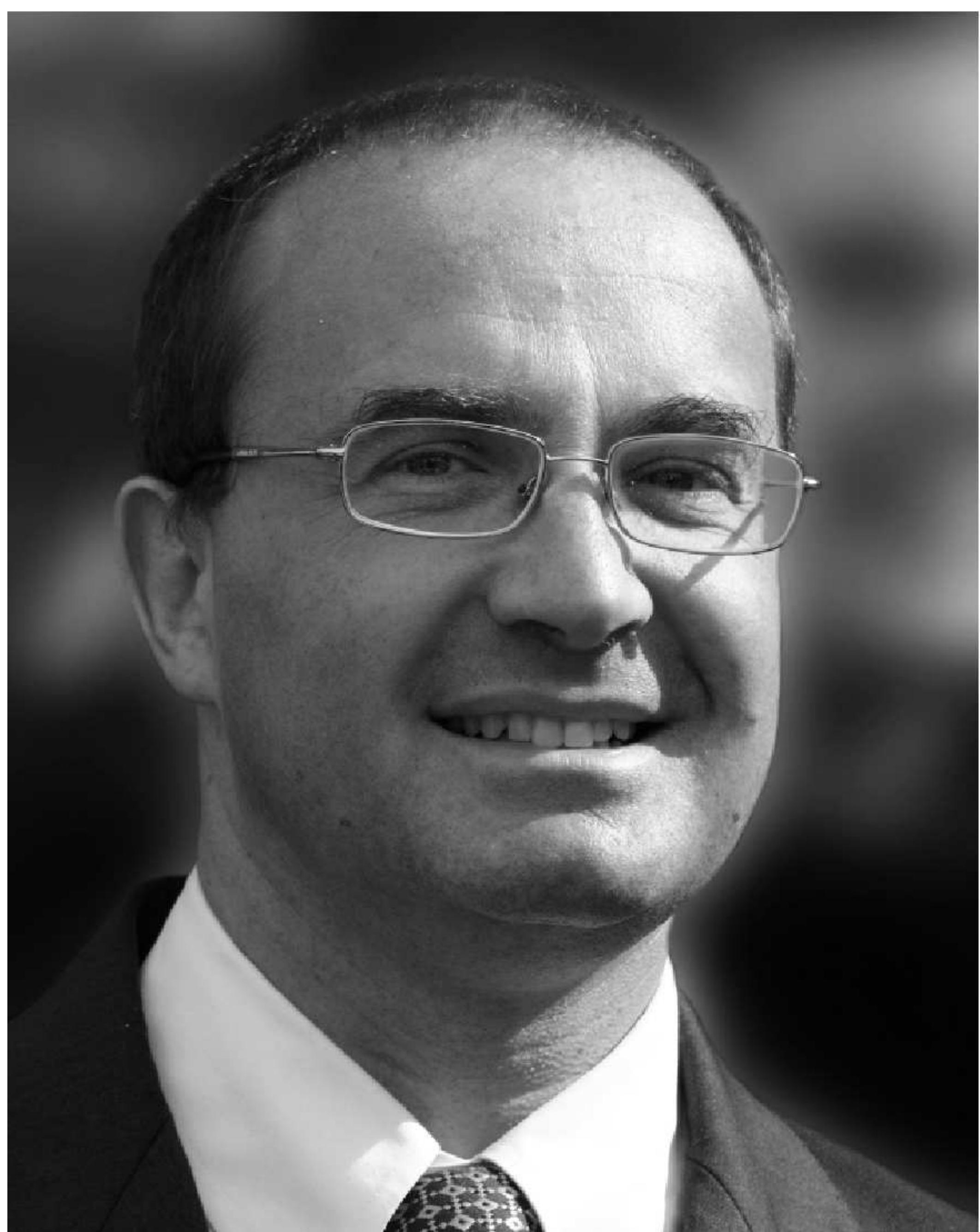




\section{Photo Daniele Munaretto}


${ }^{\star}$ Author Photo James Preisig

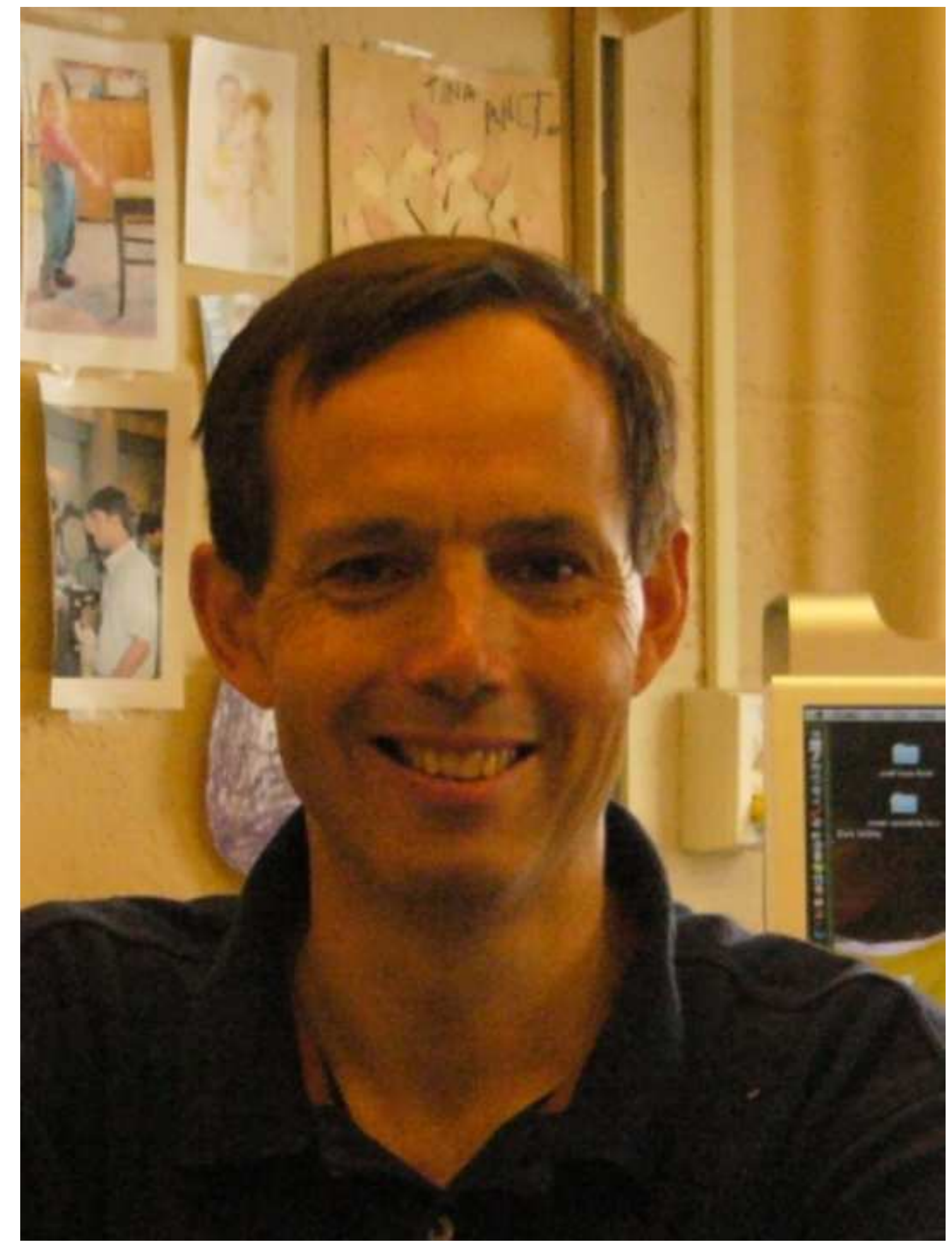




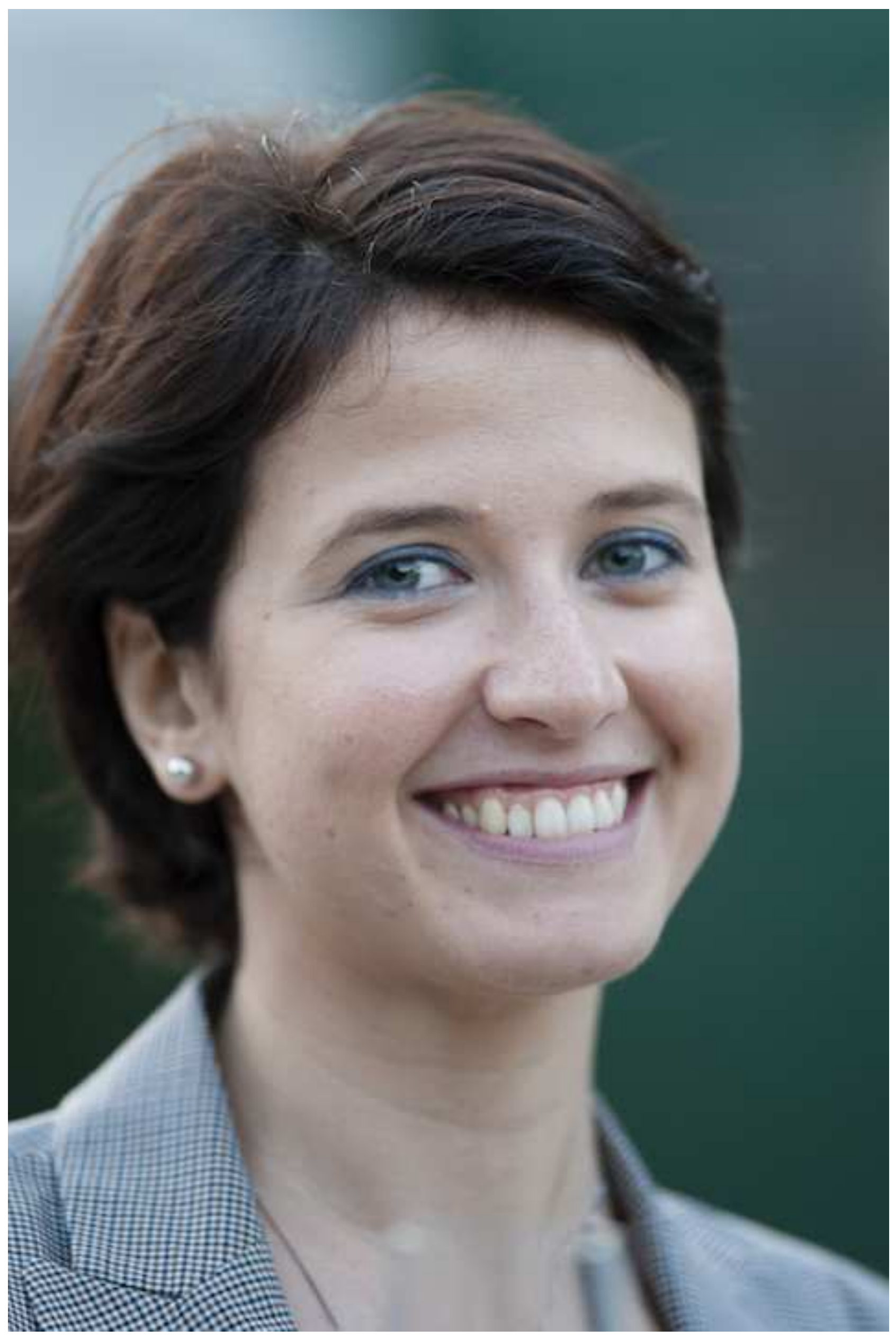

